\title{
New results for positive solutions of singular fourth-order four-point $p$-Laplacian problem
}

\author{
Minghe Pei and Libo Wang*
}

\section{"Correspondence:}

wlb_math@163.com

Department of Mathematics, Beihua

University, JiLin, 132013, P.R. China

\begin{abstract}
The existence and uniqueness of positive solutions are obtained for singular fourth-order four-point boundary value problem with $p$-Laplace operator $\left[\varphi_{p}\left(u^{\prime \prime}(t)\right)\right]^{\prime \prime}=f(t, u(t)), 0<t<1, u(0)=0, u(1)=a u(\xi), u^{\prime \prime}(0)=0, u^{\prime \prime}(1)=b u^{\prime \prime}(\eta)$, where $f(t, u)$ is singular at $t=0,1$ and $u=0$. A fixed point theorem for mappings that are decreasing with respect to a cone in a Banach space plays a key role in the proof.
\end{abstract}

MSC: 34B15; 34B16; 34B18

Keywords: $p$-Laplace operator; singular; fourth-order four-point boundary value problem; positive solution; cone

\section{Introduction}

In this paper, we investigate the existence and uniqueness of positive solutions for the singular fourth-order differential equation involving the $p$-Laplace operator

$$
\left[\varphi_{p}\left(u^{\prime \prime}(t)\right)\right]^{\prime \prime}=f(t, u(t)), \quad t \in(0,1)
$$

with the four-point boundary conditions

$$
u(0)=0, \quad u(1)=a u(\xi), \quad u^{\prime \prime}(0)=0, \quad u^{\prime \prime}(1)=b u^{\prime \prime}(\eta)
$$

where $\varphi_{p}(t)=|t|^{p-2} t, p>1,0<\xi, \eta<1,0 \leq a<1 / \xi, 0 \leq b^{p-1}<1 / \eta$, and $f(t, x)$ is singular at $t=0,1$ and $x=0$. Here by a positive solution $u$ of SBVP (1.1)-(1.2) we mean a solution $u \in C^{2}[0,1]$ with $\varphi_{p}\left(u^{\prime \prime}\right) \in C^{2}(0,1) \cap C[0,1]$ satisfying $u(t)>0$ on $(0,1)$.

It is well known that the bending of elastic beam can be described by some fourth-order boundary value problems. There are extensive studies on fourth-order boundary value problems with diverse boundary conditions by using different methods, for instance, [1$28]$ and the references therein.

Recently, in the case $0 \leq a<1,0 \leq b<1$, using the lower and upper solution method and the Schauder fixed-point theorem, Zhang and Liu [25] proved that the SBVP (1.1)-(1.2) has at least one positive solution under the following assumptions:

$\left(\mathrm{H}_{1}\right) f \in C((0,1) \times(0, \infty),[0, \infty))$, and $f(t, x)$ is nonincreasing in $x$;

(c) 2016 Pei and Wang. This article is distributed under the terms of the Creative Commons Attribution 4.0 International License (http://creativecommons.org/licenses/by/4.0/), which permits unrestricted use, distribution, and reproduction in any medium, provided you give appropriate credit to the original author(s) and the source, provide a link to the Creative Commons license, and indicate if changes were made. 
$\left(\mathrm{H}_{2}\right)$ For any constant $\lambda>0,0<\int_{0}^{1} H(s, s) f(s, \lambda s(1-s)) \mathrm{d} s<\infty$;

$\left(\mathrm{H}_{3}\right)$ There exist a continuous function $a(t)$ in $[0,1]$ and a fixed positive number $k$ such that $a(t) \geq k t(1-t), t \in[0,1]$, and

$$
\begin{aligned}
& \int_{0}^{1} G(t, r) \varphi_{p}^{-1}\left(\int_{0}^{1} H(r, s) f(s, a(s)) \mathrm{d} s\right) \mathrm{d} r:=b(t) \geq a(t), \quad t \in[0,1] \\
& \int_{0}^{1} G(t, r) \varphi_{p}^{-1}\left(\int_{0}^{1} H(r, s) f(s, b(s)) \mathrm{d} s\right) \mathrm{d} r \geq a(t), \quad t \in[0,1]
\end{aligned}
$$

where $G(t, s), H(t, s)$ will be given in Section 2.

The purpose of this paper is to improve the existence results of [25]. Using a fixed point theorem for mappings that are decreasing with respect to a cone in a Banach space, we obtain the existence and uniqueness of positive solutions of SBVP (1.1)-(1.2). We note that, in our proofs, we just assume that $\left(\mathrm{H}_{1}\right)$ and $\left(\mathrm{H}_{2}\right)$ of [25] with $\left(\mathrm{H}_{3}\right)$ of [25] removed. Our study is motivated by the papers [11,29].

In addition, we note that we also obtained the uniqueness of a positive solution for SBVP (1.1)-(1.2).

The rest of the paper is organized as follows. The fixed point theorem of Gatica et al. [29] and some definitions and lemmas are given in Section 2. The main results on the existence of positive solutions for SBVP (1.1)-(1.2) are presented in Section 3.

\section{Preliminary}

Let $B$ be a Banach space. A nonempty closed set $K \subset B$ is called a cone if the following conditions are satisfied:

(i) $a u+b v \in K$ for all $u, v \in K$ and all $a, b \geq 0$;

(ii) $u,-u \in K$ imply $u=0$.

Given a cone $K$, a partial order $\preceq$ is induced on $B$ as follows; $u \preceq v$ for $u, v \in B$ iff $v-u \in K$ (for clarity, we sometimes write $u \preceq v$ (w.r.t. $K)$ ). For $u, v \in B$ with $u \preceq v$, we denote by $\langle u, v\rangle$ the closed order interval between $u$ and $v$, that is, $\langle u, v\rangle=\{w \in B: u \preceq w \preceq v\}$. A cone $K$ is normal in $B$ if there exists $\delta>0$ such that $\left\|e_{1}+e_{2}\right\| \geq \delta$ for all $e_{1}, e_{2} \in K$ with $\left\|e_{1}\right\|=\left\|e_{2}\right\|=1$.

Lemma 2.1 ([11,29]) Let $B$ be a Banach space, $K$ a normal cone in $B, D$ a subset of $K$ such that if $u, v \in D$ with $u \preceq v$, then $\langle u, v\rangle \subset D$, and let $T: D \rightarrow K$ be a continuous mapping that is compact on any closed order interval contained in D. Suppose that there exists $u_{0} \in D$ such that $T^{2} u_{0}$ is defined and $T u_{0}$ and $T^{2} u_{0}$ are order-comparable to $u_{0}$. Then $T$ has a fixed point in $D$, provided that either

(I) $T u_{0} \preceq u_{0}$ and $T^{2} u_{0} \preceq u_{0}$ or $u_{0} \preceq T u_{0}$ and $u_{0} \preceq T^{2} u_{0}$, or

(II) the complete sequence of iterates $\left\{T^{n} u_{0}\right\}_{n=0}^{\infty}$ is defined and there exists $v_{0} \in D$ such that $T v_{0} \in D$ and $v_{0} \preceq T^{n} u_{0}$ for all $n \geq 0$.

Let $G(t, s)$ denote the Green function for

$$
-u^{\prime \prime}=0, \quad u(0)=0, \quad u(1)=\alpha u(\xi) .
$$


Then by [18] the Green function $G(t, s)$ can be expressed as follows:

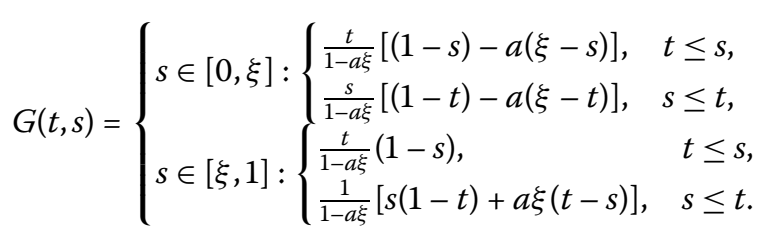

Lemma 2.2 ([25]) The Green function $G(t, s)$ has the following properties:

$$
t(1-t) G(s, s) \leq G(t, s) \leq G(s, s) \quad \text { for }(t, s) \in[0,1] \times[0,1] .
$$

Let $B=C[0,1]$ denote the Banach space of continuous functions with norm

$$
\|u\|=\sup _{t \in[0,1]}|u(t)|, \quad \forall u \in B
$$

and let $K=\{u \in B: u(t) \geq 0$ on $[0,1]\}$ be the cone of nonnegative functions in $B$. It is easy to see that $K$ is a normal cone in $B$. Now we define the subset $D \subset K$ as

$$
D:=\{u \in K: \text { there exists } \lambda(u)>0 \text { such that } u(t) \geq \lambda t(1-t) \text { on }[0,1]\} \text {. }
$$

Moreover, define $T: D \rightarrow K$ by

$$
(T u)(t):=\int_{0}^{1} G(t, \tau) \varphi_{p}^{-1}\left(\int_{0}^{1} H(\tau, s) f(s, u(s)) \mathrm{d} s\right) \mathrm{d} \tau, \quad \forall u \in D,
$$

where

$$
H(t, s)= \begin{cases}s \in[0, \eta]: \begin{cases}\frac{t}{1-b^{p-1} \eta}\left[(-s)-b^{p-1}(\eta-s)\right], & t \leq s, \\ \left.\frac{s}{1-b^{p-1} \eta}[1-t)-b^{p-1}(\eta-t)\right], & s \leq t,\end{cases} \\ s \in[\eta, 1]: \begin{cases}\frac{t}{1-b^{p-1} \eta}(1-s), & t \leq s, \\ \frac{1}{1-b^{p-1} \eta}\left[s(1-t)+b^{p-1} \eta(t-s)\right], & s \leq t .\end{cases} \end{cases}
$$

Then $T$ is well defined. In fact, from Lemma 2.2 we have

$$
t(1-t) H(s, s) \leq H(t, s) \leq H(s, s) \quad \text { for }(t, s) \in[0,1] \times[0,1] .
$$

It can be easily verified that

$$
\left|H_{t}^{\prime}(t, s)\right| \leq \begin{cases}\frac{1+b}{1-b^{p-1} \eta} s, & 0 \leq s \leq t, \\ \frac{1+b}{1-b^{p-1} \eta}(1-s), & 0 \leq t \leq s .\end{cases}
$$

It follows from conditions $\left(\mathrm{H}_{1}\right)$ and $\left(\mathrm{H}_{2}\right)$ that, for each $u \in K$,

$$
\begin{aligned}
\left|\int_{0}^{1} H_{\tau}^{\prime}(\tau, s) f(s, u(s)) \mathrm{d} s\right| \leq & \int_{0}^{1}\left|H_{\tau}^{\prime}(\tau, s)\right| f(s, \lambda s(1-s)) \mathrm{d} s \\
\leq & \frac{1+b}{1-b^{p-1} \eta}\left[\int_{0}^{\tau} s f(s, \lambda s(1-s)) \mathrm{d} s\right. \\
& \left.+\int_{\tau}^{1}(1-s) f(s, \lambda s(1-s)) \mathrm{d} s\right]:=v(\tau) .
\end{aligned}
$$


By the Fubini theorem and $\left(\mathrm{H}_{2}\right)$ it is easy to show that $v(\tau) \in L^{1}[0,1]$, and hence $\int_{0}^{1} H(\tau, s) f(s, u(s)) \mathrm{d} s \in C[0,1]$. Thus, $T$ is well defined, and also from Lemma 2.2 we have that, for all $u \in D$ and $t \in[0,1]$,

$$
\begin{aligned}
(T u)(t) & =\int_{0}^{1} G(t, \tau) \varphi_{p}^{-1}\left(\int_{0}^{1} H(\tau, s) f(s, u(s)) \mathrm{d} s\right) \mathrm{d} \tau \\
& \leq \int_{0}^{1} G(\tau, \tau) \varphi_{p}^{-1}\left(\int_{0}^{1} H(\tau, s) f(s, u(s)) \mathrm{d} s\right) \mathrm{d} \tau .
\end{aligned}
$$

Hence,

$$
\|T u\| \leq \int_{0}^{1} G(\tau, \tau) \varphi_{p}^{-1}\left(\int_{0}^{1} H(\tau, s) f(s, u(s)) \mathrm{d} s\right) \mathrm{d} \tau .
$$

On the other hand, by Lemma 2.2 , for all $u \in D$ and $t \in[0,1]$, we have

$$
\begin{aligned}
(T u)(t) & =\int_{0}^{1} G(t, \tau) \varphi_{p}^{-1}\left(\int_{0}^{1} H(\tau, s) f(s, u(s)) \mathrm{d} s\right) \mathrm{d} \tau \\
& \geq t(1-t) \int_{0}^{1} G(\tau, \tau) \varphi_{p}^{-1}\left(\int_{0}^{1} H(\tau, s) f(s, u(s)) \mathrm{d} s\right) \mathrm{d} \tau .
\end{aligned}
$$

Thus,

$$
(T u)(t) \geq t(1-t)\|T u\|, \quad t \in[0,1] .
$$

This implies that $T u \in D$, that is, $T: D \rightarrow D$, and hence, it can be verified that $u \in D$ is a positive solution of SBVP (1.1)-(1.2) iff $T u=u$.

\section{Main results}

In this section, we first establish an existence theorem of positive solutions for SBVP (1.1)(1.2) by applying Lemma 2.1 .

Before proceeding with our existence result for SBVP (1.1)-(1.2), we will define a sequence of functions that are modifications of $f$ and have none of the singularities of $f$ at $u=0$. To this end, we define a sequence of functions $f_{n}:(0,1) \times[0, \infty) \rightarrow[0, \infty)$ by

$$
f_{n}(t, u)=f(t, \max \{u, t(1-t) / n\}) .
$$

Note that, for $n=1,2, \ldots, f_{n}$ satisfies $\left(\mathrm{H}_{1}\right)$. Also, for $n=1,2, \ldots$,

$$
\begin{aligned}
& f_{n}(t, u) \leq f(t, u), \quad(t, u) \in(0,1) \times(0, \infty), \\
& f_{n}(t, u) \leq f(t, t(1-t) / n), \quad(t, u) \in(0,1) \times[0, \infty) .
\end{aligned}
$$

We now state and prove our existence result for SBVP (1.1)-(1.2).

Theorem 3.1 Assume that conditions $\left(\mathrm{H}_{1}\right)$ and $\left(\mathrm{H}_{2}\right)$ are satisfied. Then SBVP (1.1)-(1.2) has at least one positive solution $u^{*} \in D$. 
Proof We define the sequence of mappings $T_{n}: K \rightarrow K$ by

$$
\left(T_{n} u\right)(t):=\int_{0}^{1} G(t, \tau) \varphi_{p}^{-1}\left(\int_{0}^{1} H(\tau, s) f_{n}(s, u(s)) \mathrm{d} s\right) \mathrm{d} \tau, \quad \forall u \in K .
$$

Then by (3.2) and condition $\left(\mathrm{H}_{2}\right), T_{n}$ is well defined and bounded. We note that $T_{n}$ is a continuous mapping by Lebesgue's dominated convergence theorem. Also, it is easy to show by $\left(\mathrm{H}_{2}\right)$ and the continuity of $G(t, s)$ that $\left\{\left(T_{n} u\right)(t): u \in K\right\}$ is equicontinuous, and hence $T_{n}$ is a compact mapping by the Arzelà-Ascoli theorem.

In addition, observe that for all $n$ and $u \in K, T_{n} u$ satisfies the boundary conditions (1.2). Furthermore, for each $n$, since $T_{n}$ satisfies $\left(\mathrm{H}_{1}\right)$, it follows that $T_{n}$ is nonincreasing relative to the cone $K$. Also, it is clear that $0 \preceq T_{n}(0)$ and $0 \preceq T_{n}^{2}(0)$ for each $n$. Thus, by Lemma 2.1, for each $n$, there exists $u_{n} \in K$ such that $T_{n} u_{n}=u_{n}$. Hence, for each $n, u_{n}(t)$ satisfies the boundary conditions (1.2).

Now we claim that there exist $R>r>0$ such that

$$
r \leq\left\|u_{n}\right\| \leq R \text { for all } n
$$

Firstly, we shall prove the right-hand side inequality. Assume to the contrary that the inequality is false. Then by passing to a subsequence and relabeling, without loss of generality, we may assume that

$$
\lim _{n \rightarrow \infty}\left\|u_{n}\right\|=\infty \quad \text { and } \quad\left\|u_{n}\right\| \leq\left\|u_{n+1}\right\| \quad \text { for all } n .
$$

Similarly to the proof of (2.1), we can easily show that, for any $u \in K$,

$$
\left(T_{n} u\right)(t) \geq t(1-t)\left\|T_{n} u\right\|, \quad t \in[0,1] .
$$

Since $T_{n} u_{n}=u_{n}$ for each $n$, it follows that

$$
u_{n}(t) \geq t(1-t)\left\|u_{n}\right\| \geq t(1-t)\left\|u_{1}\right\|, \quad t \in[0,1] .
$$

Then assumptions $\left(\mathrm{H}_{1}\right)$ and $\left(\mathrm{H}_{2}\right)$ and inequality (3.1) yield that, for any $0 \leq t \leq 1$ and $n$,

$$
\begin{aligned}
u_{n}(t) & =\int_{0}^{1} G(t, \tau) \varphi_{p}^{-1}\left(\int_{0}^{1} H(\tau, s) f_{n}\left(s, u_{n}(s)\right) \mathrm{d} s\right) \mathrm{d} \tau \\
& \leq \int_{0}^{1} G(\tau, \tau) \varphi_{p}^{-1}\left(\int_{0}^{1} H(s, s) f\left(s, u_{n}(s)\right) \mathrm{d} s\right) \mathrm{d} \tau \\
& \leq \int_{0}^{1} G(\tau, \tau) \mathrm{d} \tau \cdot \varphi_{p}^{-1}\left(\int_{0}^{1} H(s, s) f\left(s, s(1-s)\left\|u_{1}\right\|\right) \mathrm{d} s\right):=M .
\end{aligned}
$$

Thus, $\left\|u_{n}\right\| \leq M$ for all $n$. This is a contradiction to $\lim _{n \rightarrow \infty}\left\|u_{n}\right\|=\infty$.

Next, we prove the left-hand side inequality. Assume to the contrary that the inequality is false. By passing to a subsequence and relabeling we may assume without loss of generality that $\lim _{n \rightarrow \infty}\left\|u_{n}\right\|=0$. Then we have

$$
\lim _{n \rightarrow \infty} u_{n}(t)=0 \quad \text { uniformly on }[0,1] .
$$


Since $f(t, u)$ is singular at $u=0$, by the finite covering theorem there exists $\delta>0$ such that, for $t \in[\xi / 2, \xi]$ and $0<x<\delta$, we have

$$
f(t, x)>\frac{2}{\xi(1-\xi)}\left[\int_{\xi / 2}^{\xi} H(s, s) \mathrm{d} s\right]^{-1}
$$

By (3.4) there exists $n_{0} \geq 1$ such that, for any $n \geq n_{0}$,

$$
0<u_{n}(t)<\delta / 2, \quad 0<t(1-t) / n<\delta / 2, \quad t \in[\xi / 2, \xi]
$$

Hence, for any $n \geq n_{0}$, we have

$$
\begin{aligned}
u_{n}(\xi) & =\int_{0}^{1} G(\xi, \tau) \varphi_{p}^{-1}\left(\int_{0}^{1} H(\tau, s) f_{n}\left(s, u_{n}(s)\right) \mathrm{d} s\right) \mathrm{d} \tau \\
& \geq \int_{\xi / 2}^{\xi} G(\xi, \tau) \varphi_{p}^{-1}\left(\int_{\xi / 2}^{\xi} H(\tau, s) f_{n}\left(s, u_{n}(s)\right) \mathrm{d} s\right) \mathrm{d} \tau \\
& \geq \int_{\xi / 2}^{\xi} G(\xi, \tau) \varphi_{p}^{-1}\left(\tau(1-\tau) \int_{\xi / 2}^{\xi} H(s, s) f(s, \delta / 2) \mathrm{d} s\right) \mathrm{d} \tau \\
& \geq \int_{\xi / 2}^{\xi} G(\xi, \tau) \varphi_{p}^{-1}\left(\frac{\xi(1-\xi)}{2} \int_{\xi / 2}^{\xi} H(s, s) f(s, \delta / 2) \mathrm{d} s\right) \mathrm{d} \tau \\
& \geq \int_{\xi / 2}^{\xi} G(\xi, \tau) \varphi_{p}^{-1}(1) \mathrm{d} \tau=\int_{\xi / 2}^{\xi} G(\xi, \tau) \mathrm{d} \tau=\frac{3 \xi^{2}(1-\xi)}{8(1-a \xi)},
\end{aligned}
$$

which implies $\left\|u_{n}\right\| \geq \frac{3 \xi^{2}(1-\xi)}{8(1-a \xi)}$. This is a contradiction to $\lim _{n \rightarrow \infty}\left\|u_{n}\right\|=0$.

In summary, we conclude our claim. Furthermore, from (3.3) we have

$$
r t(1-t) \preceq u_{n} \preceq R \quad(\text { w.r.t. } K), n=1,2, \ldots
$$

This implies that the sequence $\left\{u_{n}\right\}$ belongs to the closed order interval $\langle r t(1-t), R\rangle \subset D$. It is easy to see that the restriction of $T$ to $\langle r t(1-t), R\rangle$ is a compact mapping. Hence, there exists a subsequence of $\left\{T u_{n}\right\}$ that converges to some $u^{*} \in K$. Relabel the subsequence as the original sequence so that $\lim _{n \rightarrow \infty}\left\|T u_{n}-u^{*}\right\|=0$.

Also, by (3.5) there exists $n_{0}$ such that, for all $n \geq n_{0}$,

$$
t(1-t) / n \leq r t(1-t) \leq u_{n}(t) \quad \text { on }[0,1] .
$$

For all $n \geq n_{0}$ and $t \in[0,1]$, we have

$$
\begin{aligned}
\left(T u_{n}\right)(t)-u_{n}(t)= & \left(T u_{n}\right)(t)-\left(T_{n} u_{n}\right)(t) \\
= & \int_{0}^{1} G(t, \tau)\left[\varphi_{p}^{-1}\left(\int_{0}^{1} H(\tau, s) f\left(s, u_{n}(s)\right) \mathrm{d} s\right)\right. \\
& \left.-\varphi_{p}^{-1}\left(\int_{0}^{1} H(\tau, s) f_{n}\left(s, u_{n}(s)\right) d s\right)\right] \mathrm{d} \tau \\
= & 0 .
\end{aligned}
$$


This implies $\lim _{n \rightarrow \infty}\left\|T u_{n}-u_{n}\right\|=0$. It follows, in turn, that $\lim _{n \rightarrow \infty}\left\|u_{n}-u^{*}\right\|=0$, and thus $u^{*} \in\langle r t(1-t), R\rangle \subset D$ and

$$
u^{*}=\lim _{n \rightarrow \infty} T u_{n}=T\left(\lim _{n \rightarrow \infty} u_{n}\right)=T u^{*}
$$

In summary, we have $u^{*} \in D$ and $T u^{*}=u^{*}$. This completes the proof of the theorem.

Theorem 3.2 Assume that conditions $\left(\mathrm{H}_{1}\right)$ and $\left(\mathrm{H}_{2}\right)$ are satisfied. Then SBVP (1.1)-(1.2) has exactly one positive solution $u^{*} \in D$.

Proof The existence of positive solution to SBVP (1.1)-(1.2) immediately follows from Theorem 3.1. Thus, we only need to show the uniqueness.

Suppose that $u_{1}(t)$ and $u_{2}(t)$ are two positive solutions of SBVP (1.1)-(1.2). Then, by $\left(\mathrm{H}_{1}\right)$ and $\left(\mathrm{H}_{2}\right), u_{1}(t)$ and $u_{2}(t)$ are both the solutions of the following boundary value problem:

$$
\begin{aligned}
& u^{\prime \prime}(t)+\varphi_{p}^{-1}\left(\int_{0}^{1} H(t, s) f(s, u(s)) \mathrm{d} s\right)=0, \quad 0<t<1, \\
& u(0)=0, \quad u(1)=a u(\xi) .
\end{aligned}
$$

Let $w(t)=u_{1}(t)-u_{2}(t)$ on $[0,1]$. Without loss of generality, we may assume that $w(1) \geq 0$. Now we show that $w(t) \equiv 0$ on $[0,1]$. There are two cases to consider.

Case 1. $w(1)>0$. In this case, we have $w(t) \geq 0$ on $[0,1]$. Assume by contradiction that there exists $t_{0} \in(0,1)$ such that $w\left(t_{0}\right)<0$. Since $w(0)=0$ and $w(1)>0$, there exist $t_{1}, t_{2} \in$ $[0,1)$ with $t_{1}<t_{0}<t_{2}$ such that

$$
w(t)<0 \quad \text { on }\left(t_{1}, t_{2}\right), \quad w\left(t_{1}\right)=w\left(t_{2}\right)=0 .
$$

It follows that, for each $t \in\left(t_{1}, t_{2}\right)$,

$$
\begin{aligned}
w^{\prime \prime}(t) & =u_{1}^{\prime \prime}(t)-u_{2}^{\prime \prime}(t) \\
& =-\varphi_{p}^{-1}\left(\int_{0}^{1} H(t, s) f\left(s, u_{1}(s)\right) \mathrm{d} s\right)+\varphi_{p}^{-1}\left(\int_{0}^{1} H(t, s) f\left(s, u_{2}(s)\right) \mathrm{d} s\right) \leq 0 .
\end{aligned}
$$

Hence, $w(t) \geq 0$ on $\left[t_{1}, t_{2}\right]$, which is a contradiction to $w(t)<0$ on $\left(t_{1}, t_{2}\right)$. Therefore, $w(t) \geq$ 0 on $[0,1]$. Consequently, for each $t \in(0,1)$,

$$
w^{\prime \prime}(t)=-\varphi_{p}^{-1}\left(\int_{0}^{1} H(t, s) f\left(s, u_{1}(s)\right) \mathrm{d} s\right)+\varphi_{p}^{-1}\left(\int_{0}^{1} H(t, s) f\left(s, u_{2}(s)\right) \mathrm{d} s\right) \geq 0 .
$$

Thus, $w(t)$ is concave upward on $[0,1]$. Since $w(1)>0$ and $w(1)=\alpha w(\xi)$, we have $w(\xi)>0$, and hence since $0<\alpha<1 / \xi$, we have

$$
w(1)<\frac{1}{\xi} w(\xi),
$$

which is a contradiction to the upward concavity of $w(t)$ on $[0,1]$.

Case 2. $w(1)=0$. In this case, we have $w(t) \equiv 0$ on $[0,1]$. Assume to the contrary that the conclusion is false. Then, there exists $t_{0} \in(0,1)$ such that $w\left(t_{0}\right) \neq 0$. Without loss of 
generality, we may assume that $w\left(t_{0}\right)>0$. Since $w(0)=w(1)=0$, there exist $t_{1}, t_{2} \in[0,1]$ with $t_{1}<t_{0}<t_{2}$ such that

$$
w(t)>0 \quad \text { on }\left(t_{1}, t_{2}\right), \quad w\left(t_{1}\right)=w\left(t_{2}\right)=0 .
$$

It follows that, for each $t \in\left(t_{1}, t_{2}\right)$,

$$
w^{\prime \prime}(t)=-\varphi_{p}^{-1}\left(\int_{0}^{1} H(t, s) f\left(s, u_{1}(s)\right) \mathrm{d} s\right)+\varphi_{p}^{-1}\left(\int_{0}^{1} H(t, s) f\left(s, u_{2}(s)\right) \mathrm{d} s\right) \geq 0 .
$$

Since $w\left(t_{1}\right)=w\left(t_{2}\right)=0$, we have that

$$
w(t) \leq 0 \quad \text { for } t \in\left(t_{1}, t_{2}\right),
$$

which is a contradiction to $w(t)>0$ on $\left(t_{1}, t_{2}\right)$.

In summary, $w(t) \equiv 0$ on $[0,1]$. This completes the proof of the theorem.

\section{Competing interests}

The authors declare that they have no competing interests.

\section{Authors' contributions}

Both authors contributed equally to the writing of this paper. Both authors read and approved the final manuscript.

\section{Acknowledgements}

The authors thank the referee for valuable suggestions, which led to improvement of the original manuscript. This work was supported by the National Natural Science Foundation of China (11201008) and the Education Department of JiLin Province ([2016]45).

\section{Received: 27 January 2016 Accepted: 27 April 2016 Published online: 03 May 2016}

\section{References}

1. Aftabizadeh, AR: Existence and uniqueness theorems for fourth-order boundary value problems. J. Math. Anal. Appl. $116,415-426(1986)$

2. Agawal, RP: On the fourth-order boundary value problems arising in bean analysis. Differ. Integral Equ. 2, 91-110 (1989)

3. Bai, ZB, Huang, BJ, Ge, WG: The iterative solutions for some fourth-order $p$-Laplace equation boundary value problems. Appl. Math. Lett. 19, 8-14 (2006)

4. Bonanno, G, Di Bella, B: A fourth-order boundary value problem for a Sturm-Liouville type equation. Appl. Math. Comput. 217, 3635-3640 (2010)

5. Cabada, A, Ángel Cid, J, Sanchez, L: Positivity and lower and upper solutions for fourth order boundary value problems. Nonlinear Anal. 67, 1599-1612 (2007)

6. Cabada, A, Minhós, FM: Fully nonlinear fourth-order equations with functional boundary conditions. J. Math. Anal. Appl. 340, 239-251 (2008)

7. Cabada, A, Tersian, S: Multiplicity of solutions of a two point boundary value problem for a fourth-order equation Appl. Math. Comput. 219, 5261-5267 (2013)

8. Chen, $\mathrm{SH}, \mathrm{Ni}, \mathrm{W}, \mathrm{Wang}, \mathrm{CP}$ : Positive solution of fourth-order ordinary differential equation with four-point boundary conditions. Appl. Math. Lett. 19, 161-168 (2006)

9. Davis, JM, Henderson, J, Wong, PJY: General Lidstone problems: multiplicity and symmetry of solutions. J. Math. Anal. Appl. 251, 527-548 (2000)

10. Ding, $Y, X u$, J, Zhang, $X$ : Positive solutions for a $2 n$-th order $p$-positive solutions for a $2 n$ th-order $p$-Laplacian boundary value problem involving all derivatives. Electron. J. Differ. Equ. 2013, 36 (2013)

11. Eloe, PW, Henderson, J: Singular nonlinear boundary value problems for higher order ordinary differential equations. Nonlinear Anal. 17, 1-10 (1991)

12. Franco, D, O’Regan, D, Perán, J: Fourth-order problems with nonlinear boundary conditions. J. Comput. Appl. Math. 174, 315-327 (2005)

13. Graef, JR, Kong, L: Necessary and sufficient conditions for the existence of symmetric positive solutions of multi-point boundary value problems. Nonlinear Anal. 68, 1529-1552 (2008)

14. Gupta, CP: Existence and uniqueness results for the bending of an elastic beam equation at resonance. J. Math. Anal. Appl. 135, 208-225 (1988)

15. Korman, P: A maximum principle for fourth-order ordinary differential equations. Appl. Anal. 33, 267-273 (1989)

16. Lu, S, Jin, S: Existence of periodic solutions for a fourth-order p-Laplacian equation with a deviating argument. J. Comput. Appl. Math. 230, 513-520 (2009) 
17. Ma, RY, Zhang, JH, Fu, SM: The method of lower and upper solutions for fourth-order two-point boundary value problems. J. Math. Anal. Appl. 215, 415-422 (1997)

18. O'Regan, D: Solvability of some fourth (and higher) order singular boundary value problems. J. Math. Anal. Appl. 161, 78-116 (1991)

19. Ren, T, Chen, X: Positive solutions of fractional differential equation with $p$-Laplacian operator. Abstr. Appl. Anal. 2013, Article ID 789836 (2013)

20. Schroder, J: Fourth-order two-point boundary value problems; estimates by two side bounds. Nonlinear Anal. 8 , 107-114 (1984)

21. Usmani, RA: A uniqueness theorem for a boundary value problem. Proc. Am. Math. Soc. 77, 327-335 (1979)

22. Wang, Y, Liu, L, Wu, Y: Extremal solutions for $p$-Laplacian fractional integro-differential equation with integral conditions on infinite intervals via iterative computation. Adv. Differ. Equ. 2015, Article ID 24 (2015)

23. $W u$, J, Zhang, X, Liu, L, Wu, Y: Positive solution of singular fractional differential system with nonlocal boundary conditions. Adv. Differ. Equ. 2014, Article ID 323 (2014)

24. $\mathrm{Xu}, \mathrm{J}$, Yang, Z: Positive solutions for a fourth order $p$-Laplacian boundary value problem. Nonlinear Anal. 74, 2612-2623 (2011)

25. Zhang, X, Liu, L: Positive solutions of fourth-order four-point boundary value problems with $p$-Laplacian operator. J. Math. Anal. Appl. 336, 1414-1423 (2007)

26. Zhang, X, Liu, L, Wiwatanapataphee, B, Wu, Y: Positive solutions of eigenvalue problems for a class of fractional differential equations with derivatives. Abstr. Appl. Anal. 2012, Article ID 512127 (2012)

27. Zhang, X, Liu, L, Wiwatanapataphee, B, Wu, Y: The eigenvalue for a class of singular $p$-Laplacian fractional differential equations involving the Riemann-Stieltjes integral boundary condition. Appl. Math. Comput. 235, 412-422 (2014)

28. Zhao, J, Wang, L, Ge, W: Necessary and sufficient conditions for the existence of positive solutions of fourth order multi-point boundary value problems. Nonlinear Anal. 72, 822-835 (2010)

29. Gatica, JA, Oliker, V, Waltman, P: Singular nonlinear boundary value problems for second-order ordinary differential equations. J. Differ. Equ. 79, 62-78 (1989)

\section{Submit your manuscript to a SpringerOpen ${ }^{\mathcal{O}}$ journal and benefit from:}

- Convenient online submission

- Rigorous peer review

Immediate publication on acceptance

Open access: articles freely available online

- High visibility within the field

- Retaining the copyright to your article 